\title{
Ground state and constrained domain walls in Gd/Fe multilayers
}

\author{
Bas B. Van Aken* José L. Prieto $\dagger$ and Neil D. Mathur \\ Department of Materials Science and Metallurgy, \\ University of Cambridge, Cambridge, CB2 3QZ, UK
}

\begin{abstract}
The magnetic ground state of antiferromagnetically coupled Gd/Fe multilayers and the evolution of in-plane domain walls is modelled with micromagnetics. The twisted state is characterised by a rapid decrease of the interface angle with increasing magnetic field. We found that for certain ratios $M^{F e}: M^{G d}$, the twisted state is already present at low fields. However, the magnetic ground state is not only determined by the ratio $M^{F e}: M^{G d}$ but also by the thicknesses of the layers, that is the total moments of the layer. The dependence of the magnetic ground state is explained by the amount of overlap of the domain walls at the interface. Thicker layers suppress the Fe aligned and the Gd aligned state in favour of the twisted state. Whereas ultrathin layers exclude the twisted state, since wider domain walls can not form in these ultrathin layers.
\end{abstract}

PACS numbers:

The study of spin-polarised transport is key for the understanding of processes involved in spin-electronics. A particular interesting point is the effect of domain walls (DWs) on the transport properties of ferromagnetic materials, as has been shown recently. 1, 2] This interest is based on the possibility of creating sharp boundaries (high angular rotation of the magnetisation in only few atomic layers) without any non-magnetic spacer.

DWs have been studied in several systems. Domain walls can be measured in single crystal highly anisotropic thin films of $\mathrm{SrRuO}_{3}$. 3 Or between features in a patterned $\mathrm{La}_{0.7} \mathrm{Ca}_{0.3} \mathrm{MnO}_{3}$ thin film. 4] Another form of magnetic boundary has been observed in single crystalline thin films of $\mathrm{Fe}_{3} \mathrm{O}_{4}$, where at stacking faults the coupling is antiferromagnetic and $180^{\circ}$ magnetic boundaries are formed. 5] It has been shown that domain walls can be formed in Gd/FM multilayer devices, where FM is a standard ferromagnetic metal like iron, cobalt or permalloy. 6, 7] Recently, magnetoresistance measurements have been reported with current perpendicular to the in-plane DWs. 1, 2]

From the point of view of transport properties of FM metals, Gd/FM multilayers are of special interest for three main reasons: firstly the Gd/FM interface is an antiferromagnetic (AFM) sharp boundary, so it is ideal for the study of spin diffusion and spin-flip. Secondly, there is a large resistance mismatch across the interface, making the system similar to metal-semiconductor interfaces. Finally, in certain conditions as explained below, Gd/FM multilayers can support in-plane domain walls (DWs) close to the interface, so their transport properties can be studied in detail.

Current perpendicular-to-plane magnetoresistance experiments in $\mathrm{Gd} / \mathrm{Fe}$ multilayers have shown that the resistance decreases with increased applied field due to a decrease in the interface angle $\alpha_{i}$. 2] This is in qualitative agreement with magnetoresistance experiments in $\mathrm{Fe}_{3} \mathrm{O}_{4}$, where the resistance is a function of $\alpha_{i}$ controlled by the applied external field [5]. However, one of the samples in
Ref. [2] showed an upturn in the resistance at large fields and it was speculated that this is related to the formation of DWs.

The ground state domain walls at the Gd/Fe boundary have been magnetically characterised in bilayers [8] and multilayers too, but normally in quite thin layers. In these situations the interface and the AFM coupling play the most important roles in determining the magnetic structure. Traditionally, the Fe aligned state is found for $M^{F e}>M^{G d}$ and the Gd aligned state when $M^{F e}<M^{G d}$. Upon application of an external field $H$ the twisted state with DWs at the interfaces is observed. Note the distinction between $M_{s}^{G d}$ and $M^{G d} \cdot M_{s}^{G d}$ is the saturation magnetisation per volume and $M^{G d}$ is the total moment of all Gd layers together which is thickness dependent. Most articles focus on the effect of changing ratio $M_{s}^{G d}: M_{s}^{F e}$ with temperature, and therefore changing ratio $M^{F e}: M^{G d}$. Since the Curie temperature of Gd is much smaller than that of $\mathrm{Fe}, M_{s}^{G d}$ decreases rapidly with respect to $M_{s}^{F e}$ with increasing temperature.

Micromagnetic simulation has been done to gain insight in the changes in the magnetic state with applied field. We study the effect of the thicknesses $t_{F e}$ and $t_{G d}$ on the magnetic behaviour of Gd/Fe multilayers. We will show that there are distinct differences between samples with $M^{F e} \gg M^{G d}$ and samples with $M^{F e} \approx M^{G d}$. We will also show that at constant ratio $M^{F e}: M^{G d}$, and therefore the magnetic ground state is significantly influenced by the actual value of $t_{G d}$ and $t_{F e}$. We will present the phase diagram of the magnetic ground state as a function of $t_{G d}$ and $t_{F e}$. In ultra thin films the DWs on both side of the layer are not independent and interact via their surface tension. The $\mathrm{Fe}$ and $\mathrm{Gd}$ aligned state are completely suppressed for multilayers with $t_{G d}>16$ $\mathrm{nm}$ and $t_{F e}>9 \mathrm{~nm}$.

In this paper we model $\mathrm{Gd} / \mathrm{Fe}$ multilayers using the LLG micromagnetics software. [9] The modelled multilayers consist of three Fe layers, thickness $t_{F e}$, and two Gd layers with thickness $t_{G d}$. The simulation volume is 
$10 \times 10 \times t_{t o t} \mathrm{~nm}^{3}$, where $t_{t o t}$ is the total thickness of the multilayer given by $t_{t o t}=3 t_{F e}+2 t_{G d}$. The cell size in the simulation is $1 \times 1 \times 0.25 \mathrm{~nm}^{3}$, ensuring that the typical DWs found in this work are at least five to ten cells thick. In-plane magnetisation is obtained by periodic boundaries on the $x z$ and $y z$ planes. The materials parameters for $\mathrm{Fe}$ and $\mathrm{Gd}$ that have been used are listed in Table — effectively the simulations were done at $T=0 \mathrm{~K}$. The value used for $M_{s}^{G d}$ is $25 \%$ smaller than the bulk value as reported in many experimental works. 10, 11, 12] We will discuss the effect of reduced $M_{s}^{G d}$ later on. The AFM Fe-Gd interface interaction is chosen to be $A_{\text {int }}=-1.0 \mu \mathrm{erg} \mathrm{cm}^{-1}$, between the exchange coupling strengths of Fe and Gd. Furthermore, comparing work on the temperature dependence of the Co-Gd coupling and the Fe-Gd coupling indicates that $A_{\text {int }}=-1.0 \mu \mathrm{erg} \mathrm{cm}^{-1}$ is a good estimate. 13 .

TABLE I: Material parameters for Fe and Gd.

$$
\begin{aligned}
& \mathrm{Fe} \quad \mathrm{Gd} \\
& M_{s}\left(\mathrm{emu} \mathrm{cm}^{-3}\right) 1700 \quad 1508 \\
& A\left(\mu \mathrm{erg} \mathrm{cm}{ }^{-1}\right) 2.05 \quad 0.75 \\
& K\left(\mu \mathrm{erg} \mathrm{cm}{ }^{-1}\right) 1.4 \times 10^{5} 10^{2} \\
& \text { (cubic) (uniaxial) }
\end{aligned}
$$

For each simulation, a part of the hysteresis loop has been modelled from $\mu_{0} H=7.5 \mathrm{~T}$ to $\mu_{0} H=0 \mathrm{~T}$. This prevented the problem of having to choose the correct initial state at $\mu_{0} H=0 \mathrm{~T}$. The initial magnetisation was aligned with the $x$-axis and the Fe (Gd) magnetisation was parallel (antiparallel) with the positive direction. The field was applied in the positive $y$ direction. The initial field $\mu_{0} H=7.5 \mathrm{~T}$ rotates the bulk of the layers parallel with the field; the Fe $(\mathrm{Gd})$ boundary magnetisation however remains a component parallel to the positive (negative) $x$ axis. All magnetisation vectors within a single $x y$ simulation layer are identical and the $z$ component is always negligible $\left(<10^{-4} M_{s}\right)$. The angle between the magnetisation at layer depth $t$ and the applied field direction is $\phi(t)$. Thus $\phi=0^{\circ}$ corresponds to the magnetisation aligned with the $y$-axis and $\phi= \pm 90^{\circ}$ is aligned with the $x$-axis.

At low temperatures and zero field, each layer is expected to be fully FM aligned, and antiparallel with respect to the adjacent layers, creating an interface angle $\alpha_{i}=180^{\circ}$, with $\alpha_{i}=\left|\phi_{b}^{G d}\right|+\left|\phi_{b}^{F e}\right|$, where $\phi_{b}$ is the angle of the moment at the boundary layer.

The in-plane DW widths at the Gd/Fe interfaces have been calculated as shown in Fig. 11 The orientation of the moments as a function of the distance to the interface is fitted to an exponential function of the form $\phi\left(t^{\prime}\right)=$ $a \exp \left(b t^{\prime}\right)$, where $t^{\prime}$ is the distance to the interface, $a=$ $\phi\left(t^{\prime}=0\right)$ and $b$ is a fit parameter. The DW width $\delta$ is the intersection of the tangent of this function at $t^{\prime}=0$ with the $\phi=0^{\circ}$ axis.

Three magnetic states have been identified in $\mathrm{Gd} / \mathrm{Fe}$

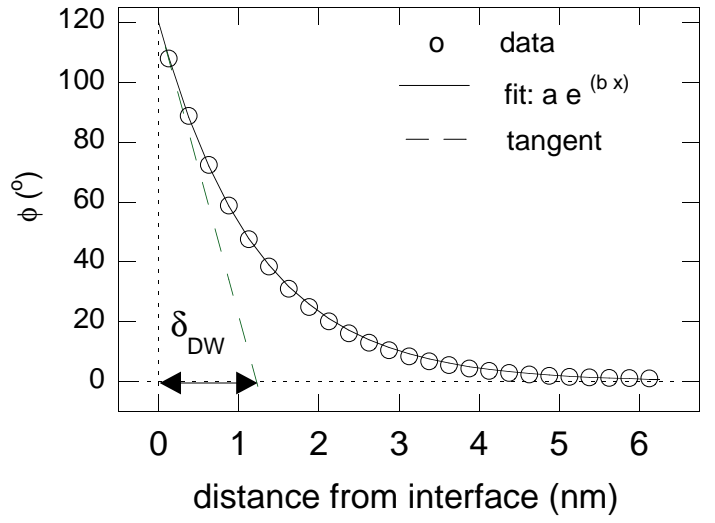

FIG. 1: Calculation of the DW width from the calculated magnetisation angle $\phi$ versus layer depth $t$ data. The drawn line is a fit to the data (o). The dashed line is the tangent at $t^{\prime}=0$, see text for explanation. The horizontal arrow indicates the DW width.

multilayers. 7, 8, 11, 14] Upon application of a small external field $H$ either the Fe layer or the Gd layer aligns with $H$. The ratio between the total magnetic moment per element $M^{G d}: M^{F e}$ determines whether the $\mathrm{Fe}$ aligned $(\mathrm{F})$ or $\mathrm{Gd}$ aligned $(\mathrm{G})$ states prevails. For instance, Hosoito et al. report for a $[\mathrm{Fe} / \mathrm{Gd}]_{15}$ multilayer film that the $\mathrm{G}$ state is present for low temperatures $T<120 \mathrm{~K}$ where $M^{G d}>M^{F e}$, and the Fe aligned state prevails for high temperatures $T>=140 \mathrm{~K}$, where $M^{G d}$ has become smaller than $M^{F e}$ since $M_{s}^{G d}$ is significantly reduced. 12] Both states are characterised by $\alpha_{i} \sim 180^{\circ}$ in small fields. A typical example of a micromagnetics simulation of the Fe aligned state is given in Fig. 2 showing a sample with $t_{F e}=10 \mathrm{~nm}$ and $t_{G d}=5 \mathrm{~nm}$. This simulation will be referred to as the $F$ state simulation in the remainder of the article.

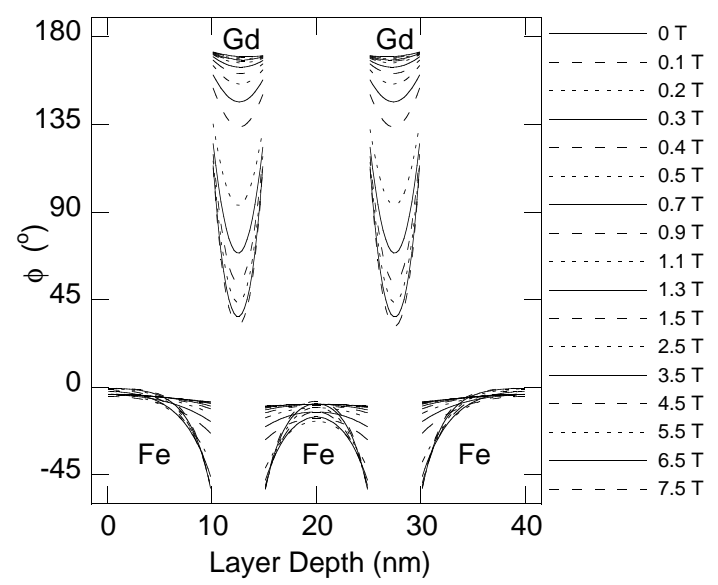

FIG. 2: Angle $\phi$ against the layer depth $t$ for the simulation with $t_{F e}=10 \mathrm{~nm}$ and $t_{G d}=5 \mathrm{~nm}$. This is an example of the $\mathrm{Fe}$ aligned state. For small $\mathrm{H}$ the magnetisation in the $\mathrm{Fe}$ layer is fully aligned with the applied external field. At large $H$ twisting occurs in the layers and $\alpha_{i}$ is reduced. 
Upon application of a large external field, most of each layer will align parallel with the field. Nevertheless, $\phi$ will be nonzero near the interfaces. This is called the twisted (T) state. Fig. 3] sketches the alignment of the layers with the applied field (parallel to the $y$-axis) and how the magnetisation rotates away in the $x y$-plane near the interfaces to accommodate the AFM coupling, thereby creating in-plane DWs.

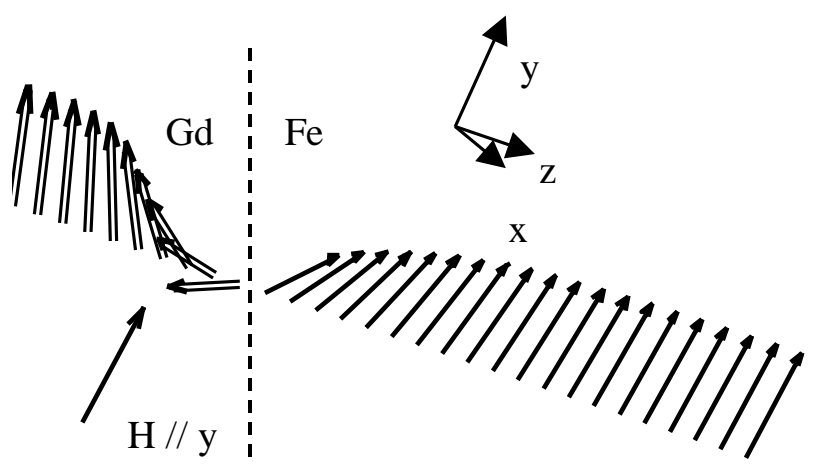

FIG. 3: Three dimensional view of the $\mu_{0} H=7.5 \mathrm{~T}$ data of Fig. 2 For clarity only the data are plotted for $12.5<t<20$ $\mathrm{nm}$, showing half of a Gd layer (thick arrows) and half of an Fe layer (thin). The magnetic field is parallel to the $y$-axis. At the interface, the Fe layer is $-45^{\circ}$ off the $y$-axis and the $\mathrm{Gd}$ layer is $+100^{\circ}$ off the $y$-axis.

The twisted state originates from the balance between the AFM coupling at the interface (that tries to align one of the layers antiparallel to $H$ ) and the Zeeman energy (which tries to align all layers parallel to $H$ ). Fig. 4 shows the twisted state simulation in a sample with $t_{F e}=15$ $\mathrm{nm}$ and $t_{G d}=20 \mathrm{~nm}$. The twisting of the FM layers is clearly present at all $H>0$. Note that the layers at the edge of the samples rotate faster, which is due to the free surface. 15] Also the high field data in Fig. 2 shows the twisted phase. The lower exchange coupling of Gd allows a larger angle between neighbouring spins, i.e. favouring narrower DWs and DWs incorporating a larger twist, compared with the Fe layer.

In experimental reports on $\mathrm{Gd} / \mathrm{Fe}$ multilayers, the saturation magnetisation of the thin Gd layers was found to be reduced by $20 \%$ to $40 \%$. 10, 11, 12] We have studied the effect of reducing $M_{s}^{G d}$ by comparing simulations of a $t_{F e}=t_{G d}=30 \mathrm{~nm}$ multilayer. Two simulations have been performed, one with $M_{s}^{G d}=2010 \mathrm{emu} \mathrm{cm}^{-3}$ corresponding to the bulk value and the other with $M_{s}^{G d}$ $=1508 \mathrm{emu} \mathrm{cm}^{-3}$ in the range of the literature values found for thin $\mathrm{Gd} / \mathrm{Fe}$ multilayers. The angles at the interface $\phi_{b}$ for both Gd and Fe layers, are extracted from the simulation and plotted in Fig. [5]

Changing $M_{s}^{G d}$ to $75 \%$ of the bulk value has only minor effects on the simulation. Experimental results indicate that the saturation magnetisation in thin Gd films might be reduced by $20 \%$ to $40 \%$. Therefore $M_{s}^{G d}$ was reduced to $75 \%$ of the bulk value in all other simulations.

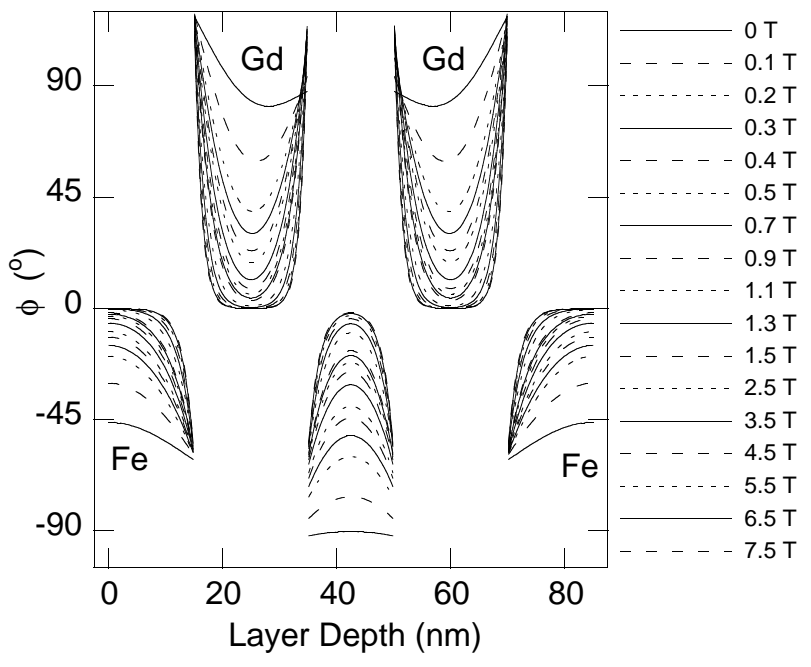

FIG. 4: $\phi$ against $t$ for the simulation with $t_{F e}=15 \mathrm{~nm}$ and $t_{G d}=20 \mathrm{~nm}$. This simulation illustrates the twisted ground state. Even at small $H \alpha_{i}$ decreases rapidly with $H$ and DWs are formed in both $\mathrm{Fe}$ and Gd layers. $\phi_{c}=0^{\circ}$ at relatively small $H$ but $\phi_{b} \gg 0^{\circ}$ for all $H$. Note that the twisting is more pronounced at low $H$ in the top and bottom layer as recently reported by Haskel et al. [15]

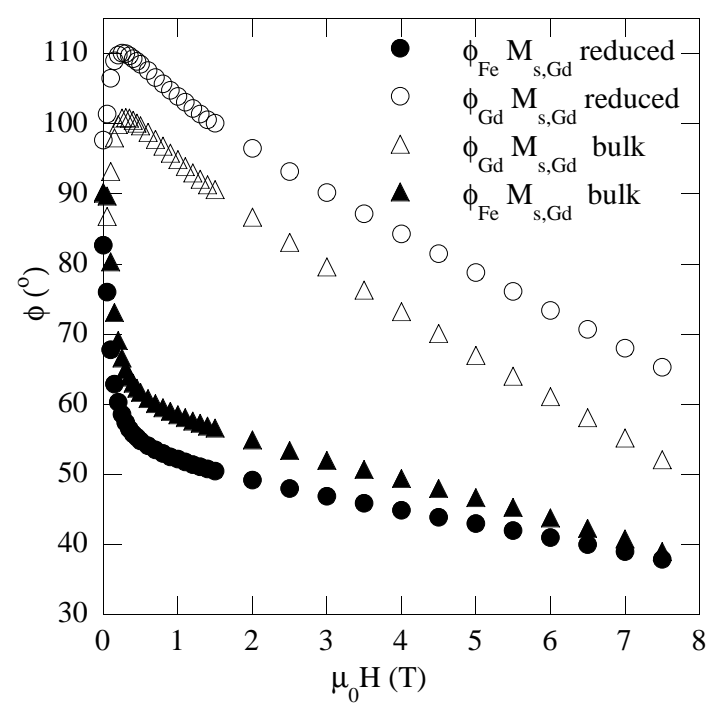

FIG. 5: Angle at the boundary $\phi_{b}$ as a function of external field. Open symbols correspond to $\phi_{b}^{G d}$ and solid symbols to $\phi_{b}^{F e}$. Circles and triangles correspond to simulations using $M_{s}^{G d}=1508 \mathrm{emu} \mathrm{cm}^{-3}$ and $M_{s}^{G d}=2010 \mathrm{emu} \mathrm{cm}^{-3}$, respectively.

We have also simulated a multilayer with a reduced interface coupling $A_{\text {int }}=-A_{G d}=-0.75 \mu \mathrm{erg} \mathrm{cm}^{-1}$. The main features, relative to the "standard" simulation with $A_{\text {int }}=-1.0 \mu \mathrm{erg} \mathrm{cm}^{-1}$, were strongly reduced $\alpha_{i}$ and boundary angles but slightly higher DW widths for all fields. However the DW width actually increased slightly with increasing field at high fields. This is related to the 
drastic decrease of $\alpha_{i}$ and the very small twists in the DWs at those fields.

Generally speaking the stronger the AFM coupling, the larger the interface angle and boundary angles. Therefore, the DWs will become narrower and have a larger twist. The narrower DWs will have less overlap and the twisted phase will still be present at smaller layer thicknesses.

We now look at the effect of sweeping the magnetic field on the magnetic state of the $\mathrm{Gd} / \mathrm{Fe}$ multilayers. We have plotted the angles $\phi_{c}, \alpha_{i}, \phi_{b}-\phi_{c}$ and DW width $\delta$ as a function of $H$ in Fig. [ 6] data is taken from the Fe aligned state simulation (Fig. (2) and twisted state simulation (Fig. (4). The main difference between the aligned states and the twisted state can be seen in the top-right panel of Fig. [6 In the twisted state simulation $\alpha_{i}$ decreases rapidly with $H$, whereas in the Fe aligned state simulation $\alpha_{i} \approx 180^{\circ}$ for small fields. The reduction of $\alpha_{i}$ with increasing field is in agreement with the experimental results reported on anti phase boundaries in $\mathrm{Fe}_{3} \mathrm{O}_{4}$ [5] and AFM coupled interfaces in Gd/Fe multilayers . 2]

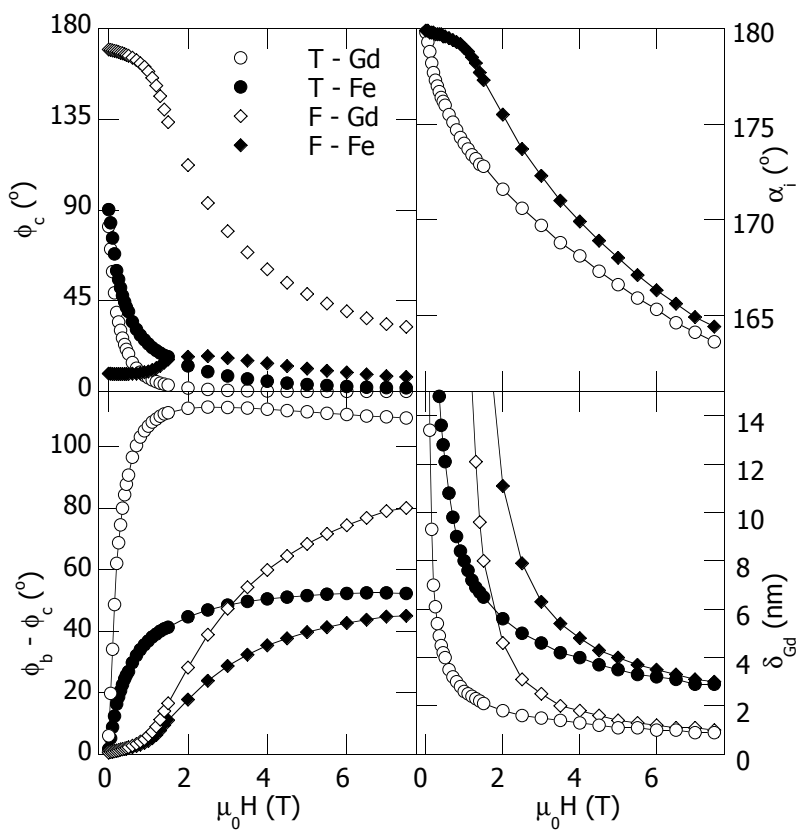

FIG. 6: Data extracted of the twisted state simulation with $t_{F e}=15 \mathrm{~nm}$ and $t_{G d}=20 \mathrm{~nm}$ and the Fe aligned state simulation with $t_{F e}=10 \mathrm{~nm}$ and $t_{G d}=5 \mathrm{~nm}$. The angle of the magnetic moment at the centre $\phi_{c}$, the interface angle $\alpha_{i}$, the twist in the DW $\phi_{b}-\phi_{c}$ and the width of the DW $\delta$ are plotted vs the applied field $H$. The figure highlights the different behaviour of the Fe and the Gd layer. Open circles ( $T$ - Gd) correspond to the Gd layer of the twisted state simulation; Closed circles ( $\mathrm{T}-\mathrm{Fe}$ ) correspond to the Fe layer of the twisted state simulation. Open diamonds (F - Gd) represent the Gd layer of the Fe aligned state simulation and closed diamonds ( F - Fe) represent the Fe layer of the Fe aligned state simulation.

The decrease of $\alpha_{i}$ in the twisted state simulation is accompanied by the formation of DWs at the interfaces of the thick layers. In the Fe aligned state simulation $\alpha_{i}$, $\phi_{b}$ and $\phi_{c}$ are only weakly dependent on the applied magnetic field. Each Gd or Fe layer remains almost perfectly aligned, i.e. no twisting in the layer. At about $\mu_{0} H=0.7$ $\mathrm{T}$, there is a change in the gradient of $\alpha_{i}, \phi_{b}$ and $\phi_{c}$ with $H$. The layers also start to twist and form wide DWs.

In both simulations we find that with increasing field the DWs become narrower and $\alpha_{i}$ decreases. The curves for $\delta_{G d}$ converge at $\mu_{o} H \sim 5 \mathrm{~T}$. Apparently, the Gd DWs in both simulations become independent of $t_{G d}$ at these high fields. The data for $\delta_{F e}$ suggest that the same will happen in the Fe layers at even higher fields $\left(\mu_{0} H>8\right.$ $\mathrm{T})$.

The samples in Fig. 6 have been simulated whilst sweeping the field from $\mu_{0} H=7.5 \mathrm{~T}$ to $\mu_{0} H=0 \mathrm{~T}$. In Fig. 7 also the increasing field sweep is shown for the Fe aligned state simulation with $t_{F e}=10 \mathrm{~nm}$ and $t_{G d}$ $=5 \mathrm{~nm}$, taking the magnetic configuration at $H=0$ as starting configuration.

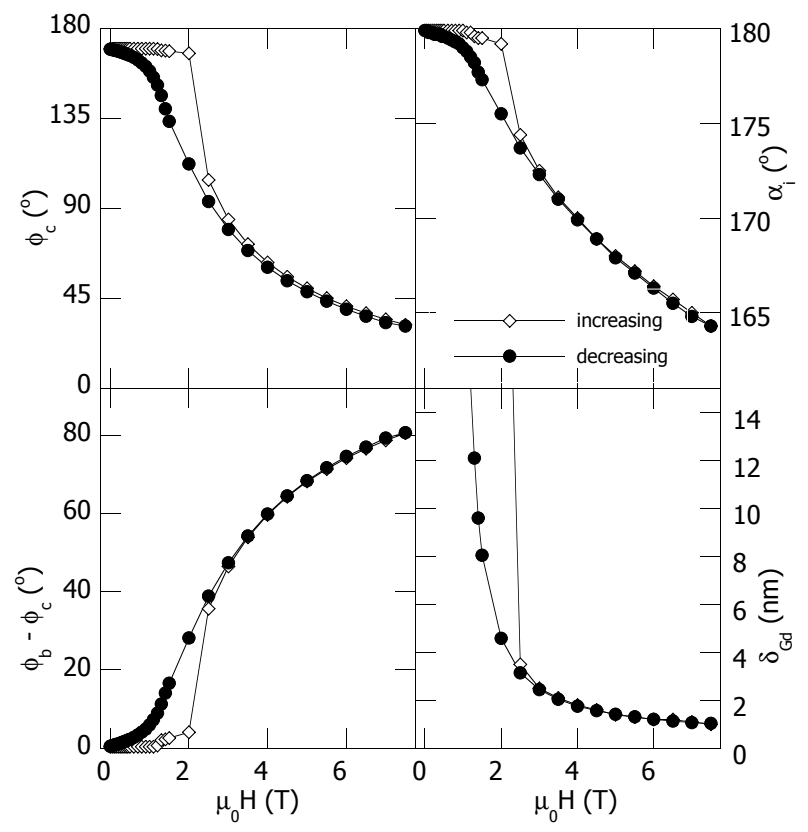

FIG. 7: Data extracted of the F aligned simulation with $t_{F e}$ $=10 \mathrm{~nm}$ and $t_{G d}=5 \mathrm{~nm}$. Data has been taken in decreasing field, followed by increasing field. Note the hysteretic behaviour at small fields. Y-axis symbols are the same as in Fig. 6

Looking at the behaviour at small fields, during the decreasing field sweep, the Fe $(\mathrm{Gd})$ layers rotate slowly but steadily to be parallel (antiparallel) with the field direction at $\mu_{0} H=0 \mathrm{~T}$. In the increasing field sweep the layers initially stay parallel just as they are at $\mu_{0} H=0$ $\mathrm{T}$. Between $\mu_{0} H=2 \mathrm{~T}$ and $\mu_{0} H=2.5 \mathrm{~T}$, the magnetic configuration abruptly changed to coincide with the decreasing field curve. Clearly the increasing field simulation got trapped in a metastable state, whereas the 
decreasing field sweep changed smoothly with $H$.

The simulation for the twisted state sample (with $t_{F e}=15 \mathrm{~nm}$ and $t_{G d}=20 \mathrm{~nm}$ ) shows no significant difference between the decreasing and increasing field sweeps. In a second twisted state simulation, after the $H \| y$ decreasing field sweep, the increasing field is parallel to $x$. $H$ is then parallel with either the Fe or the Gd magnetisation. During the increasing field sweep a similar hysteresis like transition is observed as in the $\mathrm{Fe}$ aligned state simulation. This demonstrates the importance of the initial magnetic state and the direction of the field sweep.

We have shown the typical simulations for the twisted state $\left(t_{F e}=15 \mathrm{~nm}\right.$ and $\left.t_{G d}=20 \mathrm{~nm}\right)$ and the Fe aligned state $\left(t_{F e}=10 \mathrm{~nm}\right.$ and $\left.t_{G d}=5 \mathrm{~nm}\right)$. We now investigate the effect of changing the layer thicknesses whilst keeping the ratio $M^{F e}: M^{G d}$ constant. If the magnetic configuration is only controlled by this ratio, then these simulations should exhibit qualitatively similar behaviour. In Fig. 8 we plotted as a function of $\mu_{0} H$ the data extracted for different values of $t_{F e}=t_{G d}$.

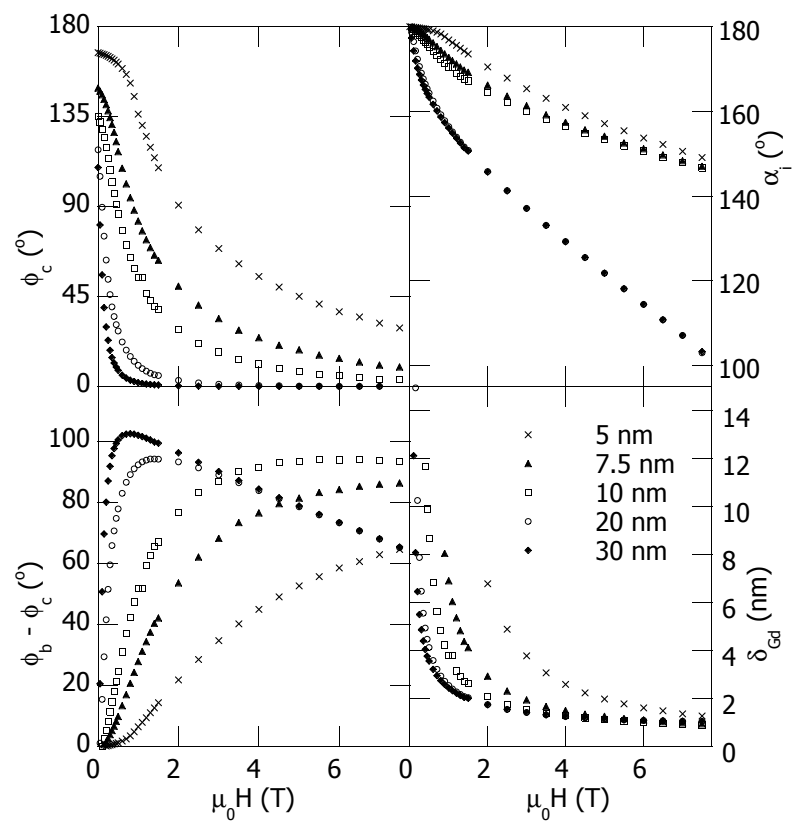

FIG. 8: The simulation of Gd/Fe multilayers for various $t_{F e}$ $=t_{G d}$. The simulations for $t_{F e}=t_{G d}=20 \mathrm{~nm}$ and $t_{F e}=t_{G d}$ $=30 \mathrm{~nm}$ are identical above $\mu_{0} H=2 \mathrm{~T}$. For clarity only the the data for the $\mathrm{Gd}$ layers are shown. Y-axis symbols are the same as in Fig. 6]

At low fields and $t_{F e}=t_{G d}<10 \mathrm{~nm}$ the $\mathrm{F}$ phase prevails, as expected since $M^{F e}>M^{G d}$ in these $t_{F e}$ $=t_{G d}$ multilayers. The prevalence of the $\mathrm{F}$ phase can be deduced from the small value of $\phi_{b}-\phi_{c}$, the slow decrease of $\alpha_{i}$, and the large value of $\phi_{c}^{G d} \gg 90^{\circ}$. Only for higher fields a transition to the twisted phase takes place and $\alpha_{i}$ starts to decrease fast. These simulations with $t_{F e}=t_{G d}<10 \mathrm{~nm}$ are similar to the Fe aligned state simulation shown in Fig. 2. However, for larger $t_{F e}=t_{G d}$ the multilayer adopts the twisted phase at all fields even though $M^{F e}>M^{G d}$. As in the twisted state simulation $\left(t_{F e}=15 \mathrm{~nm}\right.$ and $\left.t_{G d}=20 \mathrm{~nm}\right) \alpha_{i}$ is much smaller than $180^{\circ}$ at any $\mu_{0} H>0 \mathrm{~T}$ and $\phi_{b} \gg \phi_{c}$ for small fields.

The different behaviour for small and large $t_{F e}=t_{G d}$ can be explained by the overlap of DWs in the centre of each layer. For high fields $2 \delta_{G d}<<t_{G d}$ and the two DWs do not meet at the centre of the layer; they act independently. However at smaller fields (larger $\delta_{G d}$ ) and smaller $t_{G d}$ the DWs do overlap. For instance, Fig. 8 shows that at $\mu_{0} H=2.5 \mathrm{~T}$ the Gd DW is $4.8 \mathrm{~nm}$ wide for the multilayer with $t_{F e}=t_{G d}=5 \mathrm{~nm}$. The overlapping DWs reduce the twist by means of the surface tension [16] in the DW and the thin layers each become FM aligned. In Fig. 9 this scenario has been sketched. Since $M^{F e}>$ $M^{G d}$ the Fe layer will align parallel to the applied field, and $\phi_{c}^{G d}$ will approach $180^{\circ}$ at $H=0$, resulting in the $\mathrm{F}$ phase. For thicker layers, the twist is removed, i.e. the layers become totally FM aligned, only when the field is very small during a decreasing magnetic field sweep. These small fields are insufficient to rotate the Fe layers parallel with the applied field. Consequently, both layers are perpendicular to the applied field direction, $\phi_{c} \sim$ $\pm 90^{\circ}$ at $H=0$ and the twisted phase is the ground state for thicker layers.

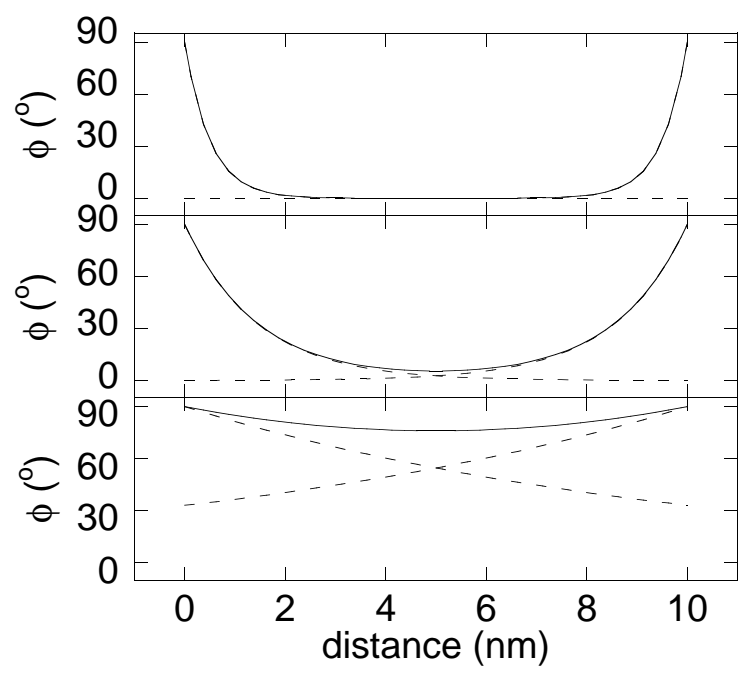

FIG. 9: Sketch of the DW overlap. The DW width is reduced from the top to the bottom panel. The surface tension of the overlapping DWs enhances the reduction of the twist.

The DW thickness depends on the layer thickness as can be observed in the bottom right corner of Fig. $8 \delta_{G d}$ increases with decreasing layer thickness at constant field. We also note that with decreasing layer thicknesses, the $H-\delta_{G d}$ curve converges at larger fields. This confirms the independent behaviour of narrow DWs in thicker layers as discussed above. 
We have shown that there is a transition from the $\mathrm{F}$ phase at $t_{F e}=t_{G d}=5 \mathrm{~nm}$ to the $\mathrm{T}$ phase at $t_{F e}=t_{G d}$ $=30 \mathrm{~nm}$, even though the ratio $M^{F e}: M^{G d}$ remained constant. Therefore, the magnetic ground state does not only depend on the ratio between the total magnetisation but also on the individual layer thicknesses. We have investigated this dependency for a range of $t_{F e}$ 's and $t_{G d}$ 's. We have chosen to use a single variable as indicator for the magnetic ground state: $\phi_{c}^{G d}$ at $H=0$. If $\phi_{c}^{G d}$ is between $45^{\circ}$ and $135^{\circ}$ the ground state of the simulation is designated as the $\mathrm{T}$ phase. Outside these boundaries either the $\mathrm{F}$ phase $\left(\phi_{c}^{G d}>135^{\circ}\right)$ or the $\mathrm{G}$ phase $\left(\phi_{c}^{G d}<45^{\circ}\right)$ is assigned. The phase diagram of the magnetic ground state as a function of $t_{F e}$ and $t_{G d}$ is plotted in Fig. 10

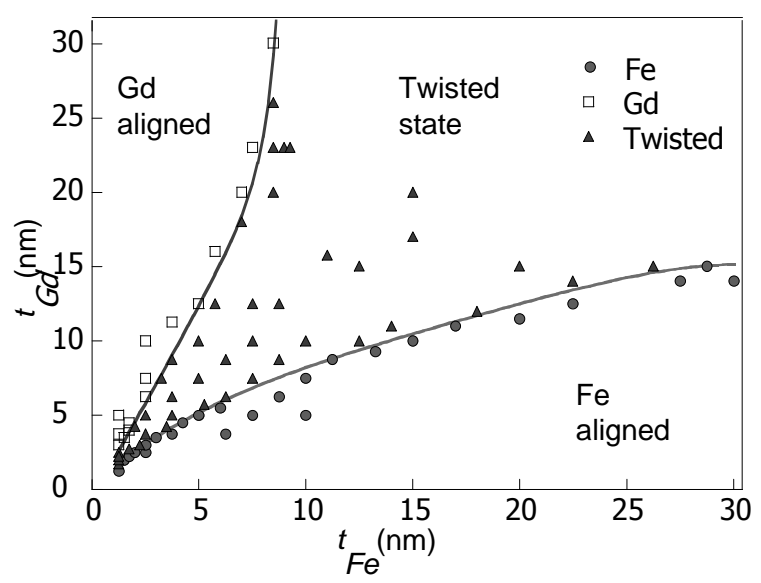

FIG. 10: Phase diagram as a function of the total Fe magnetisation (bottom axis) and the total Gd magnetisation (left axis). The drawn lines indicate the boundaries between the Gd aligned (open squares), the twisted (grey triangles) and the Fe aligned (grey circles) states. The corresponding layer thicknesses are given on the top $\left(t_{F e}\right)$ and right $\left(t_{G d}\right)$ axes.

The phase diagram confirms that the boundaries between the three magnetic ground states are not at fixed ratios of $M^{F e}: M^{G d}$. The data strongly suggests that the $\mathrm{G}$ phase is absent if $t_{F e}>9 \mathrm{~nm}$. One expects the $\mathrm{G}$ phase for multilayers with $M^{G d} \gg M^{F e}$ but the phase diagram indicates that this is only the case if $t_{F e}<9 \mathrm{~nm}$. Above this value, no matter how thick the Gd layer is, the simulation will always yield the T phase. Similarly, the $\mathrm{F}$ phase appears to be absent if $t_{G d}$ exceeds $\approx 16 \mathrm{~nm}$. We can conclude from these observations that when both layer thicknesses exceed these critical values only the $\mathrm{T}$ phase is present, independent on the ratio $M^{f e}: M^{G d}$.

Below $t_{t o t}=20 \mathrm{~nm}$, the fraction in the $\mathrm{T}$ phase drops rapidly with decreasing $t_{t o t}$. Already at high fields, the DWs overlap and reduce their twist. These thin layers do not allow the existence of DWs at small fields. This suppresses the $\mathrm{T}$ phase for ultra thin multilayers.

In conclusion, using micromagnetics $\mathrm{Gd} / \mathrm{Fe}$ multilay- ers consisting of three Fe layers and two Gd layers have been modelled to study the effect of $t_{F e}$ and $t_{G d}$ on the magnetic ground state. The twisted phase can be distinguished from the $\mathrm{Fe}$ and $\mathrm{Gd}$ aligned phases by the rapid decrease of $\alpha_{i}$ at small fields. At the same time wide DWs are formed at the edges of the thick layers. In both aligned states $\alpha_{i}$ remains almost $180^{\circ}$ in small fields. Each Gd or Fe layer remains almost perfectly aligned, i.e. no twisting in the layer. Above a threshold field, the aligned phases are transformed into the twisted phase. In all cases, at large fields the exchange constants keep $\delta_{F e}>\delta_{G d}$, with the twist in the Gd DW larger than in the Fe DW.

When the magnetic field is applied parallel with the magnetisation of one of the layers in an increasing field sweep hysteretic behaviour can be observed. The sample will show a near perfect alignment of that layer with the field and a sharp transition to the twisted state is seen.

We have found that the magnetic ground state depends both on the ratio between the total magnetisation for $\mathrm{Fe}$ and $\mathrm{Gd}$ and on the individual layer thicknesses. The different behaviour for small and large layer thicknesses can be explained by the amount of overlap of the DWs.

The phase diagram shows that independent on the ratio $M^{F e}: M^{G d}$ only the twisted phase is allowed when both layer thicknesses exceed their respective critical values. For thin layers all the magnetic ground states are allowed and the ground state found depends on the ratio $M^{F e}: M^{G d}$. Ultra thin layers do not allow the existences of DWs at small fields. This suppresses the twisted phase.

This work was supported by an E.U. Marie Curie Fellowship, the UK EPSRC and the Royal Society.

* Electronic address: aken@mbi-berlin.de Present address: Max-Born-Institut, Berlin, Germany.

$\dagger$ Present address: Dep. Fisica Aplicada, Universidad Politécnica de Madrid, Madrid, Spain.

[1] J. L. Prieto, M. G. Blamire, and J. E. Evetts, Phys. Rev. Lett. 90, 027201 (2003).

[2] J. L. Prieto, B. B. Van Aken, G. Burnell, C. Bell, J. E. Evetts, N. Mathur, and M. G. Blamire, Phys. Rev. B 69, 054436 (2004).

[3] L. Klein, Y. Kats, A. F. Marshall, J. W. Reiner, T. H. Geballe, M. R. Beasley, and A. Kapitulnik, Phys. Rev. Lett. 84, 6090 (2000).

[4] N. D. Mathur, P. B. Littlewood, N. K. Todd, S. P. Isaac, B.-S. Teo, D.-J. Kang, E. J. Tarte, Z. H. Barber, J. Evetts, and M. G. Blamire, J. Appl. Phys. 86, 6287 (1999).

[5] W. Eerenstein, T. T. M. Palstra, S. S. Saxena, and T. Hibma, Phys. Rev. Lett. 88, 247204 (2002).

[6] R. E. Camley, Phys. Rev. B 35 (1987).

[7] K. Cherifi, C. Dufour, P. Bauer, G. Marchal, and P. Mangin, Phys. Rev. B 44, 7733 (1991).

[8] O. F. K. McGrath, N. Ryzhanova, C. Lacroix, D. Givord, C. Fermon, C. Miramond, G. Saux, S. Young, and 
A. Vedyayev, Phys. Rev. B 54, 6088 (1996).

[9] M. Scheinfein, LLG Micromagnetics (1997), URL http://llgmicro.home.mindspring.com

[10] N. Ishimatsu, H. Hashizume, S. Hamada, N. Hosoito, C. S. Nelson, C. T. Venkataraman, G. Srajer, and J. C. Lang, Phys. Rev. B 60, 9596 (1999).

[11] W. Hahn, M. Loewenhaupt, Y. Y. Huang, G. P. Felcher, and S. S. P. Parkin, Phys. Rev. B 52, 16041 (1995).

[12] N. Hosoito, H. Hashizume, N. Ishimatsu, I.-T. Bae, G. Srajer, J. C. Lang, C. K. Venkataraman, and C. S. Nelson, Jap. J. Appl. Phys. 41, 1331 (2002).
[13] S. Wüchner, J. C. Toussaint, and J. Voiron, Phys. Rev. B 55, 11576 (1997).

[14] C. Dufour, K. Cherifi, G. Marchal, P. Mangin, and M. Hennion, Phys. Rev. B 47, 14572 (1993).

[15] D. Haskel, G. Srajer, Y. Choi, D. R. Lee, J. C. Lang, J. Meersschaut, J. S. Jiang, and S. D. Bader, Phys. Rev. B 67, 180406 (2003).

[16] S. Chikazumi, Physics of Ferromagnetism (Oxford University Press, 1997), chap. 16, p. 668, 2nd ed. 